Surgery for

Congenital Heart

Disease

\title{
Biventricular repair in children with atrioventricular septal defects and a small right ventricle: Anatomic and surgical considerations
}

Nilto C. De Oliveira, MD, ${ }^{a *}$ Rekwan Sittiwangkul, MD, ${ }^{a}$ Brian W. McCrindle, MD, MPH, Anne Dipchand, MD, ${ }^{\mathrm{b}}$ Tae-Jin Yun, MD, ${ }^{\mathrm{c}}$ John G. Coles, MD, ${ }^{\mathrm{a}}$ Christopher Caldarone, MD, William G. Williams, MD, ${ }^{a}$

and Glen S. Van Arsdell, MD

From the Divisions of Cardiovascular Surgery $^{\mathrm{a}}$ and Cardiology, ${ }^{\mathrm{b}}$ Hospital for Sick Children and University of Toronto, Toronto, Ontario, Canada, and the Asian Medical Center ${ }^{\mathrm{c}}$ University of Ulsan, Namgu, Ulsan City, South Korea.

Read at the Eighty-fourth Annual Meeting of The American Association for Thoracic Surgery, Toronto, Ontario, Canada, April 25-28, 2004.

Received for publication April 23, 2004; revisions received Feb 23, 2005; accepted for publication March 14, 2005.

Address for reprints: Glen S. Van Arsdell, MD, Division of Cardiovascular Surgery, Hospital for Sick Children, 555 University Ave, Toronto, Ontario, Canada M5G 1X8 (E-mail: glen.vanarsdell@ sickkids.ca).

*Present address: Department of Cardiovascular Surgery, University of Wisconsin Hospital and Clinics, Madison, Wis.

J Thorac Cardiovasc Surg 2005;130:250-7

$0022-5223 / \$ 30.00$

Copyright (c) 2005 by The American Association for Thoracic Surgery

doi:10.1016/j.jtcvs.2005.03.032
Objective: We sought to characterize morphology and to evaluate a strategy of using biventricular repair in patients with a small right ventricle and an unbalanced atrioventricular septal defect.

Methods: Thirty-eight children with a small right ventricle and an atrioventricular septal defect underwent operative therapy. Thirty-two had biventricular repair, and 6 had single-ventricle palliation. A small right ventricle was defined on the basis of echocardiographic measurements expressed as the atrioventricular valve index (ie, right atrioventricular valve/left atrioventricular valve area) and as the ratio of right ventricular to left ventricular length. Validation of measurements in patients with a small right ventricle and an atrioventricular septal defect was achieved by comparison with balanced case-matched control subjects with atrioventricular septal defects.

Results: Children with a small right ventricle and an atrioventricular septal defect had a lower atrioventricular valve index (mean, $0.41 \pm 0.1$ vs $0.52 \pm 0.1$ [control], $P<$ $.0001)$ and lower right ventricular/left ventricular length ratio $(0.78 \pm 0.1$ vs $0.99 \pm 0.17$ [control], $P<.0001)$. Children with a small right ventricle undergoing single-ventricle palliation had the smallest right ventricular dimensions. There were 4 operative deaths. Three patients with a small right ventricle and an atrioventricular septal defect who underwent biventricular repair and had an atrioventricular valve index of less than 0.50 required early reoperation for right ventricular inadequacy. A restrictive atrial fenestration was created in 11 infants. Survival at 10 years was $87 \%$ for patients undergoing biventricular repair versus $100 \%$ for control subjects $(P=.042)$.

Conclusions: Extending the use of biventricular repair in properly selected patients with a small right ventricle and an atrioventricular septal defect is feasible. An atrial fenestration improves the physiologic tolerance to repair in selected patients. The use of biventricular repair in children with atrioventricular valve indexes of less than 0.50 requires caution.

$\mathrm{T}$ he morphology of the common atrioventricular valve (CAVV) in patients with an atrioventricular septal defect (AVSD) is such that it is usually well balanced over both ventricles. However, there can be varying degrees of CAVV hypoplasia or malalignment of the ventricular septum resulting in an 
TABLE 1. Demographics, associated lesions, additional operations, intraoperative data, and clinical course

\begin{tabular}{|c|c|c|c|c|}
\hline & BVR (n = 32) & Control (n = 32) & SVP $(n=6)$ & $P$ value $^{*}$ \\
\hline \multicolumn{5}{|l|}{ Demographics } \\
\hline Median age (mo) & 6 (range, 1-47) & 6 (range, 1-58) & 8 (range, 1-58) & .81 \\
\hline Mean weight $(\mathrm{kg})$ & $5.4 \pm 2.2$ & $5.4 \pm 1.9$ & $11.3 \pm 7.5$ & .65 \\
\hline Trisomy 21 & $27(84 \%)$ & $20(62 \%)$ & $5(83 \%)$ & \\
\hline \multicolumn{5}{|l|}{ Previous operation } \\
\hline PA band & 4 & 1 & 2 & \\
\hline Coarctation & 2 & 2 & & \\
\hline PDA ligation & 1 & & & \\
\hline Cavopulmonary shunt & & & 4 & \\
\hline \multicolumn{5}{|l|}{ Associated cardiac lesions } \\
\hline Aortic coarctation & 3 & 2 & & \\
\hline PA stenosis & 4 & 2 & & \\
\hline RVOTO & 4 & 1 & & \\
\hline Muscular VSDs & 4 & 1 & & \\
\hline Moderate-severe & 4 & 3 & 1 & \\
\hline \multicolumn{5}{|l|}{ LAVVR } \\
\hline Parachute LAVV & 4 & 1 & & \\
\hline \multicolumn{5}{|l|}{ Additional operations } \\
\hline PA debanding & 4 & 1 & & \\
\hline RVMB resection & 4 & 1 & & \\
\hline PV valvotomy & 1 & & & \\
\hline PA arterioplasty & 4 & 2 & & \\
\hline Repair muscular VSDs & 3 & 1 & & \\
\hline Aortic arch repair & 1 & & & \\
\hline \multicolumn{5}{|l|}{ Intraoperative data } \\
\hline Mean CPB time & $132 \pm 44$ & $131 \pm 45$ & & .89 \\
\hline Mean crossclamp time & $94 \pm 30$ & $89 \pm 29$ & & .42 \\
\hline \multicolumn{5}{|l|}{ Clinical course } \\
\hline Median ICU days & 4 (range, 1-27) & 3 (range, 1-15) & & .23 \\
\hline Median ventilation days & 3 (range, $0-18$ ) & 1.5 (range, $0-13$ ) & & .27 \\
\hline Complete AV block & 1 & 0 & & \\
\hline Operative deaths & 3 & 0 & 1 & \\
\hline Late deaths & 1 & 0 & 1 & \\
\hline
\end{tabular}

Associated cardiac lesions are shown with the exception of patent foramen ovale and persistent ductus arteriosus. BVR, Biventricular repair; SVP, single-ventricle palliation; $P A$, pulmonary artery; $P D A$, persistent ductus arteriosus; RVOTO, right ventricular outflow tract obstruction; $V S D$, ventricular septal defect; $L A V V R$, left atrioventricular valve regurgitation; $L A V V$, left atrioventricular valve; $R V M B$, right ventricular muscle bundles; $P V$, pulmonary valve; $C P B$, cardiopulmonary bypass time (in minutes); crossclamp time, aortic crossclamp time; ICU, intensive care unit; ventilation days, mechanical ventilation days in the intensive care unit; $A V$, atrioventricular. $* P$ value (matched biventricular repair vs control groups).

unbalanced AVSD. ${ }^{1}$ Repair of a balanced AVSD is well established. ${ }^{2-6}$ Decision making and management for those with an unbalanced AVSD is challenging. More than one treatment strategy has been advocated. ${ }^{2,7,8}$

Severe hypoplasia of the left ventricle (LV) requires single-ventricle (SV) palliation. Some groups have attempted biventricular repair (BVR) for those with an AVSD and mild-to-moderate hypoplasia of the LV. ${ }^{9,10}$ An unbalanced AVSD with a small right ventricle (RV) occurs considerably less frequently than a small LV. ${ }^{9,10}$ Treatment strategies for small RV/AVSD vary and depend on interpretation of the adequacy of the RV to support a full pulmonary circulation. Few data in the literature are presented to assist in decision making. The algorithm for man- agement might include a systemic-pulmonary artery shunt, a pulmonary artery band, or a cavopulmonary anastomosis, followed by either a Fontan procedure or a 1-and-a-half ventricle repair. In some situations a conventional BVR with or without an atrial fenestration can be achieved. Difficulty exists in reliably predicting which patients will tolerate a BVR and which should have SV palliation.

Most children with a small RV/AVSD have trisomy 21. These children frequently have upper airway obstruction and a tendency to have early increased pulmonary vascular resistance. Each of these issues makes the Fontan physiology problematic. Our experience with single-ventricle palliation for patients with trisomy 21 has been challenging and disappointing. We have therefore adopted an aggressive 
TABLE 2. Morphometric variables evaluated by echocardiography $(n=70)$

\begin{tabular}{lcccc}
\hline Group & Small RV/BVR & Control/BVR & SV palliation & \multicolumn{1}{c}{$\boldsymbol{P}$ value* } \\
\hline N (patients) & 32 & 32 & 6 & $.75 \pm 0.62$ \\
RAVV area (cm ${ }^{2}$ ) & $1.70 \pm 1.14$ & $2.92 \pm 1.75$ & $2.75 \pm 1.26$ & .006 \\
LAVV area (cm $\left.{ }^{2}\right)$ & $2.58 \pm 1.28$ & $3.32 \pm 2.31$ & $0.65 \pm 0.08$ & .03 \\
AVVI (RAVV/LAVV) & $0.64 \pm 0.26$ & $0.92 \pm 0.21$ & $1.98 \pm 0.52$ & $<.001$ \\
RV length (cm) & $2.81 \pm 0.65$ & $3.98 \pm 1.30$ & $3.31 \pm 1.22$ & $<.001$ \\
LV length (cm) & $3.48 \pm 0.74$ & $3.88 \pm 1.28$ & $0.62 \pm 0.13$ & .08 \\
RV/LV length & $0.80 \pm 0.10$ & $1.03 \pm 0.13$ & $<.001$
\end{tabular}

Values are presented as means \pm standard error of the mean. $R V$, Right ventricle; $B V R$, biventricular repair; $S V$, single ventricle; $R A V V$, right atrioventricular valve area; $L A V V$, left atrioventricular valve area; $A V V I$, atrioventricular valve index; $R V$ length, right ventricular length from lateral aspect of annulus to apex; $L V$ length, left ventricular length from annulus to apex; $R V / L V$ length, right ventricle/left ventricle ratio. * $P$ value (matched biventricular repair vs control groups).

strategy for BVR in those children with a small RV/AVSD. Additionally, the intrinsic morphology of a CAVV might be prone to development of significant regurgitation, a physiologically challenging situation for the patient with Fontan physiology.

The present study evaluates outcomes after more than a decade of experience with our aggressive BVR strategy. Morphologic characteristics of patients with a small RV/AVSD are determined by comparison with case-matched control subjects.

\section{Patients and Methods}

\section{Patient Population}

Between January 1989 and July 2003, 38 children with a small RV/AVSD underwent surgical treatment at our institution. BVR

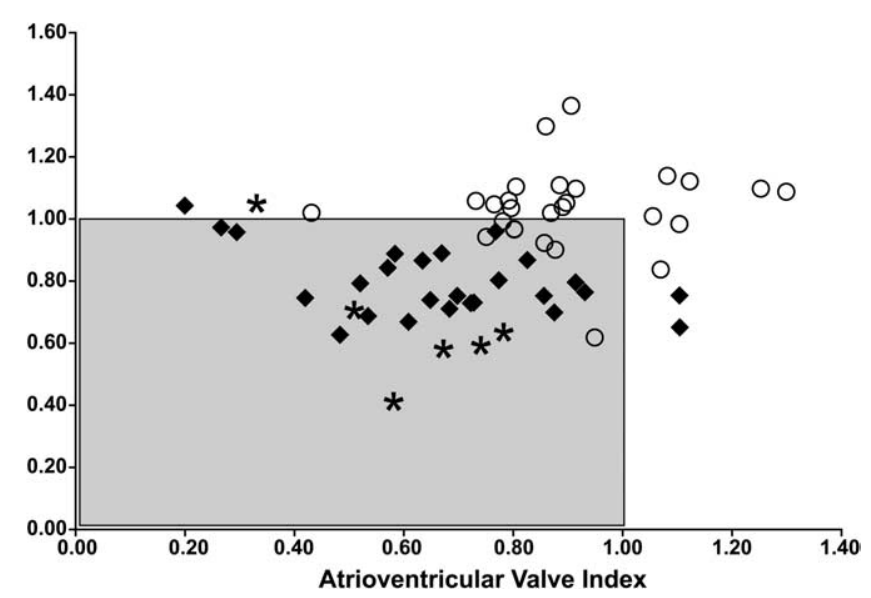

Figure 1. RV/LV length ratio and AVVI are shown in the small RV/BVR group (diamonds), the control/BVR group (circles), and the SV palliation group (asterisks). Most patients in the small RV/BVR group are within the left and inferior quadrant (rectangle) defined by an RV/LV ratio and AVVI both less than 1. Although 2 patients with a diagnosis of small RV had an AVVI of greater than 1 , they had among the lowest RV/LV ratios. Patients undergoing SV palliation had either a small AVVI or small RV/LV ratio. was accomplished in 32 (small RV/BVR group). Six children were managed with the SV palliation strategy (SV palliation group). A case-matched control group (control/BVR group) was identified and consisted of 32 children with a balanced AVSD who underwent standard BVR. The case-matched control/BVR patients met the following criteria: similar weight $( \pm 0.2 \mathrm{~kg})$, similar initial diagnosis (AVSD), and date of repair within 1 year of the matching patient with a small RV/BVR. Morphometric measurements of patients in the small $\mathrm{RV} / \mathrm{BVR}$ group were validated against the control/BVR group. Table 1 shows patient demographics, associated cardiac lesions, and procedures.

\section{Morphologic Characteristics and Echocardiographic Methodology}

Preoperative echocardiograms were analyzed in a blinded manner for all 70 patients. All measurements were made by 2-dimensional echocardiography and were the mean of 3 cardiac cycles at enddiastole. Measurements of the patients with a small RV/BVR were validated against 32 control subjects. The degree of preoperative atrioventricular valve regurgitation was evaluated by color Doppler scanning (Table 1).

A theoretical partitioning plane for the AVSD (matching the position of an AVSD repair patch) on the right side of the septum allowed for calculations of left atrioventricular valve (LAVV) and right atrioventricular valve (RAVV) areas and ratios. The subcostal view was used for calculations of CAVV area, RAVV area, and LAVV area (all in square centimeters). The atrioventricular valve index (AVVI) was calculated as the RAVV/LAVV ratio, as previously described. ${ }^{10-12}$ Ventricular dimensions were represented by ventricular length ratio (RV length [right lateral annulus to apex of the RV in centimeters]/LV length [left lateral annulus to apex of LV in centimeters]) obtained from the apical 4-chamber view.

\section{Operative Technique for Biventricular Repair}

Fifty-two (81\%) patients had an AVSD repair with our current technique: a 2-patch repair with a Dacron patch for the ventricular septal defect and glutaraldehyde-treated or fresh pericardium for the atrial component, no bridging leaflet division, and cleft closure. ${ }^{5}$ Seven $(21 \%)$ patients in the small RV/BVR group and 5 $(16 \%)$ patients in the control/BVR group had a 1-patch technique (treated pericardium patch). An atrial fenestration was used in 11 (34\%) children in the small RV/BVR group and none in the control 
TABLE 3. Reoperations after biventricular repair in children with a small right ventricle and atrioventricular septal defect

\begin{tabular}{cllclll}
\hline Patient no. & Operation & AVVI & RV/LV ratio & Reoperation & Cause of failure & Outcome \\
\hline 1 & BVR, ASD & 0.29 & 0.96 & MBTS & Severe restriction & Death on day 27 \\
2 & BVR only & 0.42 & 0.74 & BCPS & RV inadequacy & Death on day 3 \\
3 & BVR only & 0.26 & 0.98 & Creation of ASD & RV inadequacy & Alive and well \\
4 & BVR only & 0.48 & 0.62 & Creation of ASD & RV inadequacy & Alive and well \\
5 & BVR, ASD & 0.20 & 1.04 & Repair of LAVVR & Technical & Death on day 30 \\
6 & BVR only & 0.91 & 0.79 & Repair of RVSD & Technical & Alive and well \\
\hline
\end{tabular}

Values are absolute numbers for each patient. $A V V I$, Atrioventricular valve index; $R V / L V$ ratio, right ventricular length/left ventricular length ratio; $B V R$, biventricular repair; $A S D$, atrial septal defect; $M B T S$, modified Blalock-Taussig shunt; $B C P S$, bilateral cavopulmonary anastomosis; $R V$, right ventricular; $L A V V R$, left atrioventricular valve regurgitation; RVSD, residual ventricular septal defect.

group. The atrial fenestration technique evolved to an adjustable one. If a natural secundum defect is present, it is narrowed to 4 $\mathrm{mm}$. If one is not present, a 4-mm atrial septal defect (ASD) is created in the floor of the fossa ovalis or in the atrial defect patch adjacent to the limbus. A horizontal mattress suture is then placed around the ASD, with the untied ends being brought outside the heart through the intra-atrial groove or the dome of the atrium. The ASD is left open if the central venous pressure (CVP) is greater than $10 \mathrm{~mm} \mathrm{Hg}$ on defect closure or for hemodynamic instability or tachycardia. The ASD is closed by simply tying the suture outside the heart. Subtle narrowing of the ASD can also be achieved with transesophageal echocardiographic guidance. Table 1 shows intraoperative variables.

Data Acquisition, Follow-up, and Statistical Analysis The study was approved by the Institutional Review Board of the Hospital for Sick Children. Medical records and the surgical database were reviewed for demographic, operative, perioperative, and outcome data. Diagnosis and morphologic characteristics were defined by echocardiography. Additional follow-up was obtained by telephone contact to referring physicians as needed. Mean follow-up was $5.2 \pm 3.4$ and $5.1 \pm 3.0$ years in the small RV/BVR and control/BVR groups, respectively. Follow-up was $92 \%$ complete. Five patients were lost to follow-up ( 2 in the small RV/BVR group and 3 in the control/BVR group).

Data are described as frequencies, medians with ranges, and means with standard deviations as appropriate. Comparisons between characteristics of BVR group and matched control patients were made by McNemar tests, paired $t$ tests, and Wilcoxon signedrank tests. Time-related survival was determined by Kaplan-Meier methods, and comparisons between BVR group and control patients were made by log-rank tests. Correlations within the BVR group regarding echocardiographic variables and postoperative physiologic measures were made by Pearson correlation. All analyses were performed with SAS statistical software (Version 8; SAS Institute, Inc, Cary, NC).

\section{Results}

\section{Morphometric Data}

The patients in the small RV/BVR group presented with a wide spectrum of measurements, ranging from mild CAVV imbalance to severe imbalance with marked ventricular hypoplasia. By case-matched control analysis, children in the small RV/BVR group had lower mean AVVIs and mean $\mathrm{RV} / \mathrm{LV}$ length ratios when compared with patients in the control/BVR group (Table 2).

Among the small RV/BVR group, there were 3 patients in whom the AVVI was higher than the mean of the control group $(0.93,1.10$, and 1.13 , respectively); however, measurements of RV cavity size by $\mathrm{RV} / \mathrm{LV}$ ratio in these same patients were $0.77,0.66$, and 0.76 , respectively, indicating a small RV cavity size. One patient in the control/BVR group had an AVVI of 0.43, which is lower than the mean for the small RV/BVR group (Table 2). An RV/LV ratio of 1.02 in the same patient indicated balanced cavity size. The patients in the SV palliation group had the smallest RV cavity dimension (RV/LV; Figure 1). However, when compared with the small RV/BVR group, there was no difference in AVVI (Table 2).

\section{Clinical Outcomes}

The length of stay in the intensive care unit and days of mechanical ventilation were not significantly shorter in the control/BVR group than in the small RV/AVSD group (Table 1).

Six patients underwent early reoperations. Three of them died. Table 3 shows the type of reoperation, AVVI, RV/LV ratio, mechanism of failure, and outcome for reoperations in the small RV/BVR group. Four operations were for problems with RV inadequacy, and 2 of these patients survived. Of those 4 operations, 2 were for atrial fenestration creation, 1 was for ASD adjustment and creation of a systemicpulmonary artery shunt, and 1 was for a cavopulmonary anastomosis. Creation of an ASD was a successful strategy in 2 of the 3. Saturations at discharge in those 2 patients were $85 \%$ and $86 \%$, respectively. Two of the 6 reoperations were for technical failures, and 1 of these patients survived.

There were 4 hospital deaths: 3 in the small RV/BVR group (9\%), 1 in the SV palliation group (17\%), and none in the control/BVR group. Causes of failure and death, as well as associated morphometric measurements, are shown in Table 3. All deaths in the small RV/BVR group occurred in patients with an AVVI of less than 0.43. 
TABLE 4. Morphometric measures in 11 patients who underwent creation of a fenestrated atrial septal defect*

\begin{tabular}{lccccc}
\hline $\begin{array}{c}\text { Patient } \\
\text { no. }\end{array}$ & AVVI & $\begin{array}{c}\text { RV/LV } \\
\text { ratio }\end{array}$ & $\begin{array}{c}\text { Patient } \\
\text { no. }\end{array}$ & AVVI & $\begin{array}{c}\text { RV/LV } \\
\text { ratio }\end{array}$ \\
\hline 1 & 0.20 & 1.04 & 7 & 0.53 & 0.69 \\
2 & 0.29 & 0.96 & $8^{*}$ & 0.48 & 0.62 \\
3 & 0.42 & 0.74 & 9 & 0.65 & 0.74 \\
$4^{*}$ & 0.26 & 0.98 & 10 & 0.82 & 0.87 \\
5 & 0.52 & 0.79 & 11 & 0.85 & 0.75 \\
6 & 0.87 & 0.70 & & &
\end{tabular}

Values are absolute numbers for each patient. AVVI, Atrioventricular valve index; $R V / L V$ ratio, right ventricular length/left ventricular length ratio. *Patients 4 and 8 had atrial septal defect creation during the first 48 hours after biventricular repair.

One death warrants further mention. Patient 2 in Table 3 died of severe unexplained biventricular restrictive physiology after a BVR and ASD creation. Further adjustments of the ASD were attempted. A modified Blalock-Taussig shunt was performed for severe hypoxia without improvement.

In the SV palliation group only 3 of 6 patients underwent the 3 stages of palliation. Two patients underwent pulmonary artery banding $(\mathrm{PAB})$ only. One died early after the procedure, and the other had pulmonary hypertension, precluding a second-stage palliative operation, illustrating the difficulties of achieving an appropriate pulmonary artery band in children susceptible to pulmonary hypertension and upper airway obstruction. A third patient underwent $\mathrm{PAB}$ followed by a cavopulmonary shunt but is not a Fontan candidate because of pulmonary hypertension. One of the patients undergoing the Fontan operation died late of Fontan circuit thrombosis.

There was one late death in the small RV/BVR group related to complications of a noncardiac operation. No surviving patients in the small RV/BVR group have symptoms of right-heart failure. Kaplan-Meier estimates of survival were $87 \%$ at 10 years for the small RV/BVR group versus $100 \%$ for the control/BVR group $(P=.04)$.

\section{Morphometric Data and Outcome in the Small RV/BVR Group}

Four of 5 patients who demonstrated early signs of RV inadequacy and required early reoperations had AVVIs of less than 0.50 (Table 3, patients 1-4). Two of them died (patients 1 and 2), and 2 others were successfully treated with reopening of the ASD fenestration by simply dividing the suture outside the heart (in the interatrial groove) without the necessity of cardiopulmonary bypass (patient 3 and 4). Thus at a cutoff point of an AVVI of less than 0.50, there was $100 \%$ sensitivity and $95 \%$ specificity for early reoperation because of RV inadequacy and $75 \%$ sensitivity and $91 \%$ specificity for operative death. Compared with patients
TABLE 5. Evaluation of central venous pressure and arterial oxygen saturation at 6, 24, and 48 hours after the operation

\begin{tabular}{lccc}
\hline Group & Small RV/BVR & Control/BVR & $\boldsymbol{P}$ value \\
\hline $\begin{array}{l}\text { Central venous pressure } \\
6 \mathrm{~h}\end{array}$ & $12 \pm 3$ & $10 \pm 2$ & $<.05$ \\
$24 \mathrm{~h}$ & $12 \pm 4$ & $10 \pm 2$ & .13 \\
$48 \mathrm{~h}$ & $11 \pm 4$ & $11 \pm 2$ & .55 \\
Oxygen saturation & & & \\
$6 \mathrm{~h}$ & $94 \pm 6$ & $98 \pm 2$ & .003 \\
$24 \mathrm{~h}$ & $94 \pm 6$ & $98 \pm 2$ & .003 \\
$48 \mathrm{~h}$ & $94 \pm 6$ & $98 \pm 2$ & $<.01$ \\
Day of discharge & $95 \pm 3$ & $98 \pm 1$ & .008 \\
\hline
\end{tabular}

Values are presented as means \pm standard error of the mean. $R V$, Right ventricle; $B V R$, biventricular repair.

without reoperations, patients who had reoperations for RV inadequacy had smaller AVVIs $(0.36 \pm 0.1$ vs $0.69 \pm 0.2$, $P=.02)$. There was no difference in cavity size by $\mathrm{RV} / \mathrm{LV}$ ratio between the same 2 subgroups $(0.82 \pm 0.2$ vs $0.79 \pm$ $0.1, P>.2)$. Two other patients had reoperations for technical failure (patients 5 and 6 ).

\section{Morphometric Data and Use of Atrial Fenestration}

Table 4 shows the AVVI and RV/LV ratio for 11 fenestrated patients in the small RV/BVR group. Two patients had the fenestration opened in the first 48 hours after the operation (patients 4 and 8). There is a trend for a smaller annular area by AVVI measurement in the fenestrated patients.

Evaluation of CVP and Arterial Oxygen Saturation at the Initial 48 Hours After the Operation

Compared with the control/BVR group, the CVP was higher in the small RV/BVR group at 6 hours after the operation $(12 \pm 3$ vs $10 \pm 2 \mathrm{~mm} \mathrm{Hg}, P<.05)$. There was no difference at $24(12 \pm 4 \mathrm{vs} 10 \pm 2 \mathrm{~mm} \mathrm{Hg}, P=.13)$ and 48 hours $(11 \pm 4$ vs $11 \pm 2 \mathrm{~mm} \mathrm{Hg}, P=.55)$. There was no difference in CVP measurements in patients with or without atrial fenestration at 6,24 , and 48 hours after surgical intervention. In the small RV/BVR group there was no correlation between CVP measurements and AVVI or $\mathrm{RV} / \mathrm{LV}$ ratio during the initial 48 hours (Table 5).

Compared with control patients, the small RV/BVR group had a lower oxygen saturation at 6,24, and 48 hours and at hospital discharge (all values, $P<.005$; Table 5). In the small RV/BVR group there was no correlation between oxygen saturation and AVVI or RL/LV ratio at 6, 24, and 48 hours and the day of hospital discharge. Patients with atrial fenestration had lower oxygen saturations at 24 hours (mean, $90 \%$ vs $98 \%, P=.003$ ). 


\section{Discussion}

Attempts at establishing morphologic criteria for BVR in AVSD associated with a small LV have been made. It has been noted that the size of the LV might be underestimated if septal deviation is not taken into consideration. ${ }^{10}$ Van Son and colleagues ${ }^{10}$ estimated potential LV volume by taking ventricular septal bowing into consideration and determined that a preoperative potential $\mathrm{LV}$ volume of $15 \mathrm{~mL} / \mathrm{m}^{2}$ was compatible with adequate LV output after surgical intervention. Accurately predicting RV volumes is not possible with standard echocardiographic techniques, thereby making evaluation of the adequacy of the RV volume difficult. Cohen and associates, ${ }^{9}$ in a morphometric analysis of unbalanced AVSD, used RAVV and LAVV ratios to define an AVVI. A recommendation for SV palliation was made for patients with a small LV and an AVVI of less than 0.67. Only 3 of their 26 of patients had a small RV. No conclusions could be drawn about the small RV/AVSD. We attempted to morphologically define patients with a small RV/AVSD and to correlate those findings with clinical and physiologic outcomes.

\section{Morphometric Data and Echocardiographic Diagnosis} In the present study patients with a small RV/AVSD were first defined by an echocardiographic impression. Validation of that impression was achieved by a case-matched control group of patients having a balanced AVSD. We have attempted to deal with the problems of measurement of the RV cavity by a combination of AVVI and RV/LV length ratio. Significant differences in morphology were identified between the case-matched control patients and the patients in the small RV/AVSD group, thereby validating the initial echocardiographic impression of a small RV. Importantly, 3 patients in the small RV/AVSD group had an AVVI greater than the mean of the control group. In each of these 3 patients, the RV/LV length ratio was between 0.66 and 0.76 (ie, approximately 2 standard deviations smaller than the control value of $1.03 \pm 0.13$ ). It might be imputed that functional capacity of the small RV importantly has 2 components: an inlet size and a cavity size.

\section{Morphometric Data and Outcome}

The lower limit either of AVVI or RV size (RL/LV) for which a BVR for small RV/AVSD can be successfully performed is not known. In our study all patients with AVVIs of greater than 0.50 survived the operation without clinical signs of RV failure. Although our sample size is small, all 4 patients who demonstrated early signs of RV inadequacy and required early reoperations had an AVVI of less than 0.50 .

There was a correlation between reoperation for RV inadequacy and a small AVVI but no correlation between reoperation and $\mathrm{RV} / \mathrm{LV}$ ratio.
The 3 operative deaths in the small RV/BVR group occurred in patients with severe CAVV imbalance (AVVI $<0.43$ ). The fact that the 2 who survived had successful urgent reopening of atrial fenestrations suggests that prophylactic use of a fenestration in those with an AVVI of less than 0.50 might be beneficial, irrespective of immediate postrepair physiology.

Patients in the SV palliation group had quantitatively the smallest cavity sizes demonstrated by the lowest RV/LV ratios; however, because of the small sample size (only 6 patients), no conclusion could be drawn from this group.

Use of Fenestration ASD in the Small RV/BVR Group We have used a fenestrated ASD in 11 (34\%) patients in this series ( 2 early reoperations included). Two patients who had the fenestration closed in the operating room had early signs of right heart failure and were successfully treated with reopening of the ASD fenestration. These patients had good hemodynamics and right-sided filling pressures of $12 \mathrm{~mm}$ $\mathrm{Hg}$. Through the early recovery phase, RV compliance diminished, necessitating removal of the externalized ASD closure suture. Cardiopulmonary bypass was not necessary. Our need to reopen some atrial fenestrations in the first night has led to a more liberal use of an adjustable fenestration. We now leave a fenestration for those patients having small RV/AVSD if their early CVP after separation from cardiopulmonary bypass is $10 \mathrm{~mm} \mathrm{Hg}$ or greater. We would also leave a fenestrated ASD in all cases of an AVVI of less than 0.50 .

No statistical correlation was established between AVVI, $\mathrm{RV} / \mathrm{LV}$ ratio, and the use of atrial fenestration. A possible explanation is the adapted strategy of more liberal use of an atrial fenestration. It is clear, however, that the reopening of a fenestration in the early postoperative period was a lifesaving maneuver in 2 patients (Table 4 , patients 5 and 6 ).

\section{Measurements of CVP and Oxygen Saturations}

Compared with control patients, only the small RV/BVR group had a higher early postoperative CVP at 6 hours after surgical intervention. This finding suggests that most patients in the small RV/BVR group maintained adequate cardiac output during the initial postoperative period. Hemodynamic instability in this period is likely best managed by atrial fenestration or fenestration enlargement rather than creation of a cavopulmonary shunt. Early in our experience, one child had creation of a cavopulmonary shunt. Failure of the cavopulmonary shunt to achieve hemodynamic instability might be related to too high pulmonary vascular resistance and to addition of a significant operation to a critically unstable child.

Measurements of oxygen saturation during the initial 48 hours were lower in the small RV/BVR group because of the presence of a fenestrated ASD in 10 patients. As a 
subgroup, the fenestrated patients had the lowest oxygen saturations.

\section{Limitations of the Study}

Numerous variables play a role in the clinical outcome of patients. This was an evolving strategy. Decisions made early in the experience were sometimes different than what current decisions would be. There were some children with small RVs managed with an SV palliation strategy who might have been treated with BVR and one patient in the small RV/BVR group who might have benefited from an atrial fenestration rather than a bidirectional caval pulmonary shunt. The sample size and number of events prevents meaningful statistical analysis of predicted success of BVR as a correlation to AVVI and RV/LV ratio. One is then left with morphologic markers of successful repair that can be tested for RV adequacy on the basis of hemodynamics and saturation at separation from cardiopulmonary bypass. Because of sample size, the presented data are useful but not definitive.

\section{Conclusions}

Our experience demonstrates that extending the use of BVR in properly selected patients having small RV/AVSD is feasible. Both the ventricular cavity size (RV/LV ratio) and the magnitude of CAVV imbalance (AVVI) are helpful to accurately assess the potential for BVR. BVR was successful in infants having an AVVI as small as 0.50 and an $\mathrm{RV} / \mathrm{LV}$ ratio as small as 0.66 . The use of BVR in children with an AVVI of less than 0.50, on the basis of our experience, should be used with caution. If attempted, an atrial fenestration should be used at initial repair. Finally, the use of an adjustable atrial fenestration might extend the anatomic limits for BVR and improve the safety of repair.

\section{References}

1. Bharati S, Lev M. The spectrum of common atrioventricular orifice (canal). Am Heart J. 1973;86:553-61.

2. Studer M, Blackstone EH, Kirklin JW, Pacifico AD, Soto B, Chung GK, et al. Determinants of early and late results of repair of atrioventricular septal (canal) defects. J Thorac Cardiovasc Surg. 1982;84: 523-42.

3. Hanley FL, Fenton KN, Jonas RA, Mayer JE, Cook NR, Wernovsky $\mathrm{G}$, et al. Surgical repair of complete atrioventricular canal defects in infancy: twenty-year trends. J Thorac Cardiovasc Surg. 1993;106: 387-97.

4. Bando K, Turrentine MW, Sun K, Sharp TG, Ensing GJ, Miller AP, et al. Surgical management of complete atrioventricular septal defects: a twenty year experience. J Thorac Cardiovasc Surg. 1995;110:154354.

5. Fortuna RS, Ashburn DA, De Oliveira NC, Burkhart HB, Konstantinov IE, Coles JG, et al. Atrioventricular septal defects: effect of bridging leaflet division on early valve function. Ann Thorac Surg. 2004;77:895-902.

6. Tweddell JS, Litwin SB, Berger S, Friedberg DZ, Thomas JP, Frommelt PC, et al. Twenty-year experience with repair of complete atrioventricular septal defects. Ann Thorac Surg. 1996;62:419-24.
7. Freedom RM, Bini RM, Rowe RD. Endocardial cushion defect and significant hypoplasia of the left ventricle: a distinct clinical and pathological entity. Eur J Cardiol. 1978;7:263-81.

8. Clapp SK, Perry BL, Farooki ZQ, Jackson WL, Karpawich PP, Hakimi $\mathrm{M}$, et al. Surgical and medical results of complete atrioventricular canal: a ten year review. Am J Cardiol. 1987;59:454-8.

9. Cohen MS, Jacobs ML, Weinberg PM, Rychik J. Morphometric analysis of unbalanced common atrioventricular canal using two-dimensional echocardiography. J Am Coll Cardiol. 1996;28:1017-23.

10. Van Son JAM, Phoon CK, Silverman NH, Hass GS. Predicting feasibility of biventricular repair of right-dominant unbalanced atrioventricular canal. Ann Thorac Surg. 1997;63:1657-63.

11. Chin AJ, Yeager SB, Sanders SP, Williams RG, Bierman FZ, Burger $\mathrm{BM}$, et al. Accuracy of prospective two-dimensional echocardiographic evaluation of left ventricular outflow tract in complete transposition of the great arteries. Am J Cardiol. 1985;55:759-64.

12. Silverman NH. Atrioventricular septal defects (atrioventricular canal defects). In: Silverman NH, editor. Pediatric echocardiography. Baltimore, (MD): Williams \& Wilkins; 1993. p. 142-66.

\section{Discussion}

Dr Giovanni Stellin (Padova, Italy). I congratulate you and your unit for the excellent results in this difficult group of patients.

I have 3 questions for you. First, you have considered AVVI and ventricular length ratio in assessing the degree of RV hypoplasia. Indeed, although the AVVI is a solid and well-established method to assess atrioventricular valve orifice size, ventricular length ratio is not a well-validated method to assess RV volume. Would you like to give us some more details on how you have been guided by the 2 methods in judging the RV capability to bear a full cardiac output?

Dr De Oliveira. Thank you for your comments and question. This is a retrospective study. On the basis of the echocardiographic diagnosis and surgical impression, we selected a group of patients with a small RV and an unbalanced AVSD and who underwent biventricular repair. Our goal was to define morphometric measurements to facilitate the surgical decision.

The AVVI is widely accepted by surgeons and cardiologists as a measurement of valve area and balance. The use of the linear dimension to evaluate ventricular size is questionable, and in this respect I agree with you.

There is no perfect method to evaluate the ventricular volume or cavity size in these patients. Magnetic resonance imaging is being used to evaluate ventricular volume in hypoplastic left heart syndrome and might become useful in the near future, but it is expensive, it requires general anesthesia, and it is only available in major centers.

In our study the AVVI was a predictor of outcome, but we could not show any statistical significance with RV/LV ratio or linear dimension.

Dr Stellin. Second question: You have had 3 deaths among 38 patients with a borderline RV BVR, and 2 of them died of early postoperative RV failure. Plus you had to return 2 patients to the operating room to perforate the atrial septum because of early RV failure and low output syndrome. All these patients had an AVVI of less than 0.5. Do you think that in your cohort of patients with a small RV you might be able to identify a subgroup with an AVVI of less than 0.5 than might be better treated with a bilateral Glenn procedure in association with the repair, perhaps anticipated by main PAB?

Dr De Oliveira. Among the 3 deaths in the 5 patients who had AVVIs of less than 0.5 , one patient had low output syndrome and 
died from severe LAVV regurgitation caused by a tear in the superior bridging leaflet after an attempted repair. A second patient had severe restrictive cardiomyopathy of unclear cause, and a third patient earlier in the series had severe right heart failure and died after an emergency bicaval pulmonary anastomosis in an attempt to unload the RV. The other 2 patients were hemodynamically stable in the operating room, with CVPs of less than $10 \mathrm{~mm} \mathrm{Hg}$ and during the first 48 hours had severe right heart failure with low output syndrome. They improved dramatically after emergency reopening of the ASD and left the hospital without further complications.

Our technique of adjustable ASD is simple. We create a fenestration in the floor of the fossa ovalis about $4 \mathrm{~mm}$ in diameter and close it with a horizontal mattress suture with the knot outside the heart over the intra-atrial groove. The fenestration can be reopened by simply cutting the suture and can be done in the intensive care unit without the need for cardiopulmonary bypass. What was your question regarding the use of $\mathrm{PAB}$ in preparation for a one-and-a-half ventricle repair?

Dr Stellin. With an index of less than 0.5 .

Dr De Oliveira. Yes, in borderline patients. It is a valid strategy, and that is something that we should explore in the future, but our results with PAB in the AVSD in the early 1980s were dissatisfying.
In addition, in this study, of 6 patients in the SV palliation tract, only 3 had a complete Fontan operation. Three others died or had pulmonary hypertension after PAB. If we had a reliable way to protect the pulmonary circulation other then $\mathrm{PAB}$, perhaps some medication some day, then that would probably be a very valid option. In small series from Liverpool, good results have been obtained after one-and-a-half ventricle repair in 9 patients with small RV AVSDs. However, those patients had RV outflow tract obstruction and consequently a protected pulmonary circulation to start with.

Dr Stellin. Third question: As you have pointed out in your presentation, the most important limiting factor for a BVR is a small right atrioventricular valve annulus. In repairing these patients, have you have considered the possibility of borrowing some CAVV tissue to enlarge the RAVV annulus because the size of the annulus is always a very important limiting factor for a BVR?

Dr De Oliveira. This is also a good question. We have not looked at that specifically, but I know that in the past 2 years, there are patients in whom we tried to modify the orientation of the patch to gain area on the hypoplastic side, but I cannot specifically tell how many patients in this group had that particular technique used. 